\title{
Anderson's "Theorem" and Bogoliubov-de Gennes Equations for Surfaces and Impurities
}

\author{
K. Tanaka and F. Marsiglio, ${ }^{\text {a }}$ \\ a Department of Physics, University of Alberta, Edmonton, Alberta, Canada T6G 2J1
}

In order to incorporate spatial inhomogeneity due to nonmagnetic impurities, Anderson [纤 proposed a BCStype theory in which single-particle states in such an inhomogeneous system are used. We examine Anderson's proposal, in comparison with the Bogoliubov-de Gennes equations, for the attractive Hubbard model on a system with surfaces and impurities.

The procedure for examining surface and impurity effects on a microscopic level is by now well established. One uses a mean field-like decoupling, with potentials which are determined from self-consistency requirements. These potentials are then used in the effective Hamiltonian, which is numerically diagonalized. This process is continued until self-consistency is achieved. This is the essence of the Bogoliubov-de Gennes (BdG) 2] formalism.

An earlier proposal was suggested by Anderson [1], in which the single-particle problem is first diagonalized. Eigenvalues and eigenstates are obtained, with which one can formulate the BCS problem, but in a vector space associated with these eigenstates. In certain situations, the single-particle problem can be obtained analytically (open boundaries, for example [3]), or it can be obtained numerically with significantly less effort than required by the full BdG process. In these instances it would be advantageous to utilize the Anderson prescription. In this paper we report on some test cases to evaluate the Anderson prescription.

The BdG equations are well documented [2]. In this work we utilize the attractive Hubbard model, with open boundaries, and with the possibility for single site impurity potentials. The resulting equations are 酒:

$$
E_{n} u_{n}(\ell)=\sum_{\ell^{\prime}} A_{\ell \ell^{\prime}} u_{n}\left(\ell^{\prime}\right)+\Delta_{\ell} v_{n}(\ell)
$$

$E_{n} v_{n}(\ell)=-\sum_{\ell^{\prime}} A_{\ell \ell^{\prime}} v_{n}\left(\ell^{\prime}\right)+\Delta_{\ell}^{*} u_{n}(\ell)$

where

$A_{\ell \ell^{\prime}}=-t \sum_{\delta}\left(\delta_{\ell^{\prime}, \ell-\delta}+\delta_{\ell^{\prime}, \ell+\delta}\right)+\delta_{\ell \ell^{\prime}}\left(V_{\ell}-\mu+\epsilon_{\ell}\right)$

The self-consistent potentials, $V_{\ell}$, and $\Delta_{\ell}$, are given by

$$
\begin{gathered}
\Delta_{\ell}=|U| \sum_{n} u_{n}(\ell) v_{n}^{*}(\ell)\left(1-2 f_{n}\right) \\
V_{\ell}=-|U| \sum_{n}\left[\left|u_{n}(\ell)\right|^{2} f_{n}+\left|v_{n}(\ell)\right|^{2}\left(1-f_{n}\right)\right],
\end{gathered}
$$

where $|U|$ is the strength of the attractive interaction, the index $n$ labels the eigenvalues (there are $2 N$ of them), the index $\ell$ labels the sites ( 1 through $\mathrm{N}$ ), and the composite eigenvector is given by $\left(\begin{array}{l}u_{n} \\ v_{n}\end{array}\right)$, of total length $2 N$. The sums in Eqs. (1.5) are over positive eigenvalues only. The other physical parameters are the single-particle hopping, $t$, the single site impurity potentials, $\epsilon_{\ell}$, and the chemical potential, $\mu$. The $f_{n}$ is the Fermi function, with argument $\beta E_{n}$, where $\beta \equiv \frac{1}{k_{B} T}$, with $T$ the temperature. The single site electron density, $n_{\ell}$, is given, through Eq. (5), by $V_{\ell}=-|U| \frac{n_{\ell}}{2}$.

These equations are iterated to convergence, with results to be presented below.

The alternative Anderson formalism [1] first solves for the eigenvalues and eigenstates of the 
'non-interacting' problem, i.e.,

$E_{n}^{0} w_{n}(\ell)=\sum_{\ell^{\prime}} A_{\ell \ell^{\prime}}^{0} w_{n}\left(\ell^{\prime}\right)$,

where

$A_{\ell \ell^{\prime}}^{0}=-t \sum_{\delta}\left(\delta_{\ell^{\prime}, \ell-\delta}+\delta_{\ell^{\prime}, \ell+\delta}\right)-\delta_{\ell \ell^{\prime}}\left(\mu-\epsilon_{\ell}\right)$

The $N \times N$ matrix equation (6) is solved for its eigenvalues $E_{n}^{0}$ and eigenvectors $w_{n}$. This amounts to determining the unitary matrix $U_{\ell n}$ that gives a basis for the electron operators

$c_{\ell \sigma}^{\dagger}=\sum_{n} U_{\ell n}^{*} \tilde{c}_{n \sigma}^{\dagger}$,

which diagonalizes the single-particle Hamiltonian. From this matrix we determine the transformed electron-electron interaction:

$V_{n m, n^{\prime} m^{\prime}}=-|U| \sum_{\ell} U_{\ell n}^{*} U_{\ell m}^{*} U_{\ell n^{\prime}} U_{\ell m^{\prime}}$,

which now mediates the (generally off-diagonal) electron-electron interaction. The gap and number equations are derived in the usual way; they are in general complicated - the gap is a function of the quantum label $n$ and the chemical potential is shifted by an $n$-dependent quantity. Once these are obtained, we can transform back to real space, and examine the gap function or the electron density, for example, as a function of position.

Figure 1 illustrates the gap parameter obtained by the BdG formalism as a function of position, for all densities, in the case of open boundary conditions $(\mathrm{OBC})$, in one dimension. Results in higher dimension will be very similar [5]. Variations in the gap are strongest near the boundaries, as expected, and the Anderson prescription is reasonably accurate in reproducing the oscillations (not shown). In Fig. 2 we show the gap as a function of position for the case of a single impurity (at site 16) with a repulsive potential, with periodic boundary conditions (PBC). As expected, the gap is suppressed at this site, and once again, the Anderson prescription semiquantitatively reproduces the BdG result.

\section{REFERENCES}

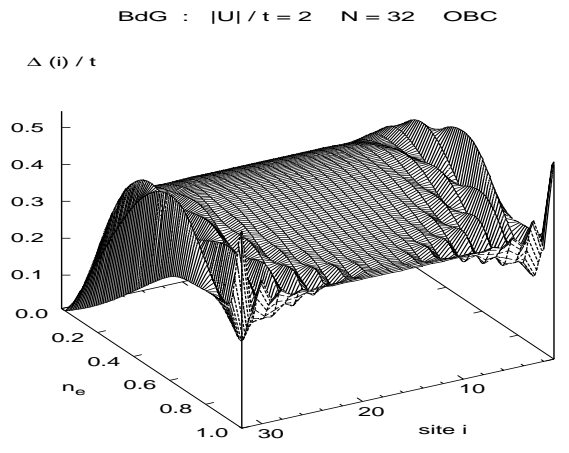

Figure 1. $\Delta(i)$ for all densities $n_{e}(\mathrm{OBC})$.

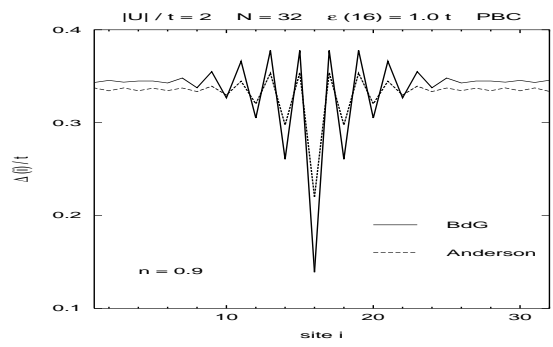

Figure 2. $\Delta(i)$ for $n_{e}=0.9(\epsilon(16)=1.0 t)$.

1. P. W. Anderson, J. Phys. Chem. Solids 11, 26 (1959).

2. P. G. de Gennes, Superconductivity of Metals and Alloys (W.A. Benjamin, Inc. New York, 1966).

3. We thank Stuart Trugman for pointing out to us that a tight-binding analytical solution exists for this problem.

4. For a tight-binding formulation of the BdG equations, see J.E. Hirsch, Physica C 194119 (1992).

5. K. Tanaka and F. Marsiglio, unpublished. 